\title{
Cochrane Eyes and Vision: a perspective introducing Cochrane Corner in Eye
}

\author{
Jennifer Evans $\mathbb{D}^{1} \cdot$ Tianjing $\mathrm{Li}^{2} \cdot$ Gianni Virgili $^{3} \cdot$ Richard Wormald ${ }^{4} \cdot$ on behalf of Cochrane Eyes and Vision
}

Received: 9 October 2018 / Accepted: 12 October 2018 / Published online: 19 February 2019

(c) The Royal College of Ophthalmologists 2019

\begin{abstract}
In 1972, Archie Cochrane wrote "It is surely a great criticism of our profession that we have not organised a critical summary, by specialty or subspecialty, adapted periodically, of all relevant randomised controlled trials". The Cochrane Collaboration arose in response to Archie Cochrane's challenge. Cochrane Eyes and Vision aims to prepare and promote access to systematic reviews of interventions for preventing or treating eye conditions and/or visual impairment, and helping people adjust to visual impairment or blindness. To identify all relevant randomised controlled trials, Cochrane Eyes and Vision has a team of information specialists who develop search strategies to identify studies for inclusion in Cochrane reviews. Since 1997 we have published 266 protocols, 193 new reviews and 158 updated reviews. The majority of these are reviews of intervention effectiveness; three reviews are diagnostic test accuracy reviews. Overall $18 \%$ of reviews contain no trials, highlighting a potential evidence gap. We provide training, education and guidance to systematic review authors and work with clinical and patient partners to prioritise and disseminate reviews. In addition, Cochrane Eyes and Vision US satellite carries out critical methodologic research addressing topics relevant to producing high-quality reviews. We are partnering with the journal Eye to publish commentaries on selected Cochrane systematic review findings. This partnership will allow us to make high-quality evidence available to ophthalmologists and other practitioners, researchers, policy makers and patients.
\end{abstract}

\section{Background to Cochrane}

In 1972, Archie Cochrane wrote "It is surely a great criticism of our profession that we have not organised a critical summary, by specialty or subspecialty, adapted periodically, of all relevant randomised controlled trials" [1]. The Cochrane Collaboration arose as a response to Archie Cochrane's challenge. Iain Chalmers set out the principles

$\triangle$ Jennifer Evans

jennifer.evans@1shtm.ac.uk

1 International Centre for Eye Health, London School of Hygiene and Tropical Medicine, Keppel Street, London WC1E 7HT, UK

2 Department of Epidemiology, Johns Hopkins University Bloomberg School of Public Health, 615 North Wolfe Street, Baltimore, MD 21205, USA

3 Department of Translational Surgery and Medicine, Eye Clinic, Via le Morgagni 85, University of Florence, 50134 Florence, Italy

4 Moorfields Eye Hospital NHS Foundation Trust, City Road, London EC1V 2PD, UK behind the Cochrane Collaboration in 1993 [2]. He highlighted the fact that it is difficult for people making decisions about health care to find all relevant information and they must rely on reviews of the evidence. Chalmers argued that reviews occupy a key link between research and improved outcomes of health care and that they should be conducted using scientific principles and regularly updated.

Chalmers recognised that the only way to achieve this huge task was to make collaboration a key feature of the effort. At an early stage, the Cochrane Collaboration was organised around the principle of the "collaborative review group", a group of individuals with a shared interest in a particular area of health care. Another important early decision was to publish the work of the Cochrane Collaboration electronically, thus making it easier to keep each review up to date and to ensure that any errors can be corrected quickly.

The Cochrane Collaboration has driven the development of methods for evidence synthesis [3]. In particular, risk of bias tools for randomised controlled trials [4], nonrandomised studies [5] and the use of GRADE to assess 
the certainty of the evidence [6] with incorporation of summary of findings tables with input from users of the information [7]. The methods used in Cochrane reviews are summarised in the Cochrane Handbook and are freely available online (training.cochrane.org/handbook). Cochrane has also developed a core set of methodological expectations based on the Cochrane handbook (methods. cochrane.org/mecir). As a result of this international collaborative activity on methods, Cochrane reviews are usually judged to be higher quality than other reviews $[8,9]$.

\section{Cochrane Eyes and Vision}

The Cochrane Eyes and Vision collaborative review group was registered in 1997. Cochrane Eyes and Vision contributes systematic reviews of the effects of interventions for visual impairment and eye disease to The Cochrane Library (www.eyes-cochrane.org). Box 1 presents an explanation of the key features of a systematic review. We have contributors from all around the world with over 1000 authors and 400 peer reviewers predominantly from the UK, USA, China, Australia, Italy, Brazil, Mexico and India.

Cochrane Eyes and Vision identifies the evidence, provides training and education to people who undertake systematic reviews (the clinical community and others), provides guidance to authors undertaking systematic reviews, and works with clinical and patient partners to prioritise and disseminate reviews. The editorial base for the group is located at the London School of Hygiene and Tropical Medicine (LSHTM) in the UK and is funded by the UK National Institute for Health Research (NIHR). The UK editorial base is responsible for the publication of Eyes and Vision reviews in The Cochrane Library.

The group has two satellites. The Cochrane Eyes and Vision US satellite, located at Johns Hopkins Bloomberg School of Public Health in Baltimore, Maryland, has been funded by the National Eye Institute of the National Institutes of Health since 2002. The overall objective of the US satellite is to develop a critical mass of US-based vision researchers and practitioners who are trained in preparing and using systematic reviews as part of Cochrane Eyes and Vision. In addition to identifying the evidence, and

Box 1 What is a systematic review?

"A Systematic Review is a review of a clearly formulated question that uses systematic and reproducible methods to identify, select and critically appraise all relevant research, and to collect and analyse data from the studies that are included in the review. A systematic review can be either quantitative or qualitative." http://libguides.library.curtin.edu.au/c.php?g=202420\&p= 1332858 educating and guiding the workforce, the US satellite focuses on providing a centre for comparative effectiveness research and performs methodological research needed to improve the quality of systematic reviews.

The Diagnostic Test Accuracy (DTA) satellite is based at the University of Florence, Italy. The DTA satellite provides specific support for DTA reviews which have a different structure compared with intervention reviews and are more complex to conduct. They require specific statistical and methodological tools and, above all, a discussion on the clinical pathway and the decision context where the test under investigation is used. The largest DTA review published by the satellite includes 106 studies investigating the accuracy of imaging tests for diagnosing manifest glaucoma [10].

Cochrane has recently developed networks of CRGs. Cochrane Eyes and Vision is in the Musculoskeletal, Oral, Skin and Sensory network (https://www.cochrane. org/about-us/our-global-community/review-group-netw orks).

\section{Cochrane Eyes and Vision output 1997-2018}

\section{Identifying the evidence}

Cochrane Eyes and Vision has a team of information specialists who develop search strategies to identify studies for inclusion in Cochrane reviews. A register of reports of randomised controlled trials (RCTs) is maintained and is available by searching the Cochrane Central Register of Controlled Trials (CENTRAL) in The Cochrane Library. We also search specific journals manually to identify additional reports of RCTs and these results are added to CENTRAL. Cochrane has developed a machine-learning classifier to identify RCTs [11]. CEV information specialists assess the ophthalmic-related results from the classifier and provide feedback on the accuracy of the search algorithm.

\section{Reviews and protocols}

The first Cochrane Eyes and Vision review was published in 1998 and examined the role of antioxidant vitamins and minerals in the progression of age-related macular degeneration and included three randomised trials. The 10th update of this review was published in 2017 [12] and now includes 20 published trials. Since 1997 we have published 266 protocols, 193 new reviews and 158 updated reviews (Table 1). The majority of these reviews are reviews of intervention effectiveness; three reviews are diagnostic test accuracy reviews. Overall 18\% of reviews contain no trials, highlighting a potential 
Table 1 Cochrane Eyes and Vision new reviews, updated reviews and protocols

\begin{tabular}{lcccccc}
\hline Year & Protocols & New reviews & Updated reviews & $\begin{array}{l}\text { Most recent update } \\
\text { of review }\end{array}$ & $\begin{array}{c}\text { Empty reviews } \\
\text { (latest update) }\end{array}$ & $\begin{array}{l}\text { Median (range) studies } \\
\text { included }^{\mathrm{b}}(\text { latest update) }\end{array}$ \\
\hline $1997-2004$ & $64(24 \%)$ & $27(14 \%)$ & $12(8 \%)$ & $1(0.5 \%)$ & 0 & 8 \\
$2005-2009$ & $77(29 \%)$ & $50(26 \%)$ & $35(22 \%)$ & $17(9 \%)$ & $2(12 \%)$ & $4(1-53)$ \\
$2010-2014$ & $84(32 \%)$ & $50(26 \%)$ & $75(47 \%)$ & $74(38 \%)$ & $16(22 \%)$ & $4(1-42)$ \\
$2015-2018$ & $41(15 \%)$ & $66(34 \%)$ & $36(23 \%)$ & $101(52 \%)$ & $16(16 \%)$ & $6(1-137)$ \\
Total & $266(100 \%)$ & $193(100 \%)$ & $158(100 \%)$ & $193(100 \%)$ & $34(18 \%)$ & $5(1-137)$ \\
\hline
\end{tabular}

${ }^{a}$ As a percentage of total number of reviews

${ }^{\mathrm{b}}$ Excluding empty reviews

evidence gap. The median number of trials included in non-empty reviews is 5 (range 1-137).

\section{Methods research}

Since 2002, Cochrane Eyes and Vision US satellite has carried out critical methodologic research related to systematic reviews. This research has engaged clinical partners and addresses topics highly relevant to producing highquality reviews. Investigators at Cochrane Eyes and Vision US satellite have published methods papers on prioritising reviews [13-16], practice guidelines [17-19], identifying clinical trials [20], strengths and limitations of data sources [21-24], and outcomes in Eyes and Vision trials and reviews [25-29], dissemination by co-publication [30], and network meta-analysis methods as applied to open-angle glaucoma [31, 32].

\section{Cochrane Eyes and Vision partnerships}

\section{Academic partnerships including guideline panels}

Cochrane Eyes and Vision US satellite has worked with the American Academy of Ophthalmology to update their preferred practice guidelines [18, 19] and partnered with other professional organisations, such as American Academy of Optometry and American Optometric Association. The US satellite has provided collaborative educational programmes hosted by a number of academic institutions and pioneered the concept of Cochrane Eyes and Vision Centre for Evidence-based Vision care accreditation for medical centres undertaking Cochrane reviews. The Cochrane Eyes and Vision UK editorial base had a formal collaboration with the National Institute for Health and Care Excellence (NICE). As part of this collaboration, we produced a series of review and review updates within a timeframe to support the production of their guidelines on macular degeneration and cataract. We also have links with the Royal College of Ophthalmologists and College of Optometrists.

\section{Patients}

Cochrane Eyes and Vision works with individuals affiliated with patient and consumer groups. These patient and consumer partners have the opportunity to participate in prioritising questions to be addressed by our systematic reviews. They also contribute to developing systematic review protocols and the systematic reviews themselves, specifically in ensuring that the questions asked and the outcomes that matter to them are addressed. Patient partners also serve as peer reviewers, providing their comments to reviews before publication. An important role for patient contributors is to advise on appropriate language to be used in plain language summaries, contributing to social media exchanges.

\section{Eyes and Vision journals}

Cochrane Eyes and Vision has partnered with Eyes and Vision journals to increase the quality of systematic reviews published in traditional journals. The model was put in place in 2011, when Tianjing Li was appointed as an associate editor for Systematic Reviews for Ophthalmology. The Editor-in-Chief (Dr. George Bartley) and Dr. Li developed guidance for authors submitting such papers, and have instituted new quality standards. Similar agreements have now been reached with the American Journal of Ophthalmology, BMC Ophthalmology, Current Eye Research, Eye, JAMA Ophthalmology, Journal of Glaucoma, Retina, Ophthalmic Epidemiology, Ophthalmology Glaucoma, Optometry and Vision Science.

\section{Dissemination}

Cochrane Eyes and Vision disseminates its reviews by working with guideline panels co-ordinated by NICE, 
American Academy of Ophthalmology, Royal College of Ophthalmologists and the College of Optometrists. We encourage review author teams co-publish their Cochrane reviews in other journals [30] or write articles on the implications of the reviews [33]. We prepare a plain language summary for each review and a visual summary or blogshot (https://eyes.cochrane.org/blogshots) along with other teaching materials, such as slidesets. Going forward, we will be editing the relevant Wikipedia page once each review is published. Our review author teams are encouraged to involve patients and the public in their reviews. Cochrane has an active consumer network (https:// consumers.cochrane.org/). We are active on social media (@ cochraneeyes). Cochrane Eyes and Vision US satellite is evaluating novel dissemination techniques, such as creating 3-5 minute YouTube videos. Each video describes the results of a Cochrane Eyes and Vision review using plain language narratives and animated graphics.

\section{Cochrane Corner in Eye}

If health-care providers and patients are to make wellinformed decisions about their vision care, then we must produce systematic reviews of the evidence with all due speed. Cochrane Eyes and Vision aims to prepare and promote access to systematic reviews of interventions for preventing or treating eye conditions and/or visual impairment, and helping people adjust to visual impairment or blindness. As part of our dissemination activities, we have teamed up with Eye and will be publishing a "Cochrane Corner" at regular intervals in the journal. This will consist of an abstract of a published review with an invited clinical commentary on the implications of the review. We are excited about the opportunity of publishing summaries of Cochrane systematic review findings in the journal Eye. This partnership will allow us to make high-quality evidence available for a broad range of patients, practitioners, researchers and policy makers. It will provide an important route to ensure that the findings of our reviews are disseminated to clinicians making decisions about eye health care.

Acknowledgements Thank you to all the contributors to Cochrane Eyes and Vision particularly those working at the editorial base, editors (https://eyes.cochrane.org/cev-editorial-base) and satellites (https://eyes.cochrane.org/cev-us-satellite; https://eyes.cochrane. org/cev-italian-dta-satellite).

Funding Cochrane Eyes and Vision is funded by a Cochrane Infrastructure Grant from the National Institute of Health Research (NIHR). Cochrane Eyes and Vision US satellite is funded by NIH grant UG1EY020522 (PI: Tianjing Li, MD, MHS, PhD). Richard Wormald is funded by financial support from the Department of Health through the award made by the NIHR to Moorfields Eye Hospital NHS Foundation Trust and UCL Institute of Ophthalmology for a Specialist Biomedical
Research Centre for Ophthalmology. The views expressed in this publication are those of the authors and not necessarily those of the funders.

\section{Compliance with ethical standards}

Conflict of interest The authors declare that they have no conflict of interest.

Publisher's note: Springer Nature remains neutral with regard to jurisdictional claims in published maps and institutional affiliations.

\section{References}

1. Cochrane A. Effectiveness and efficiency. Random reflections on health services. London: Nuffield Provincial Hospitals Trust, 1972.

2. Chalmers I. The Cochrane collaboration: preparing, maintaining, and disseminating systematic reviews of the effects of health care. Ann N Y Acad Sci. 1993;703:156-63.

3. Chandler J, Hopewell S. Cochrane methods-twenty years experience in developing systematic review methods. Syst Rev. 2013;2:76 https://doi.org/10.1186/2046-4053-2-76.

4. Higgins JP, Altman DG, Gotzsche PC, et al. The Cochrane Collaboration's tool for assessing risk of bias in randomised trials. BMJ. 2011;343:d5928 https://doi.org/10.1136/bmj.d5928.

5. Sterne JA, Hernan MA, Reeves BC, et al. ROBINS-I: a tool for assessing risk of bias in non-randomised studies of interventions. BMJ. 2016;355:i4919 https://doi.org/10.1136/bmj.i4919.

6. Guyatt GH, Oxman AD, Vist GE, et al. GRADE: an emerging consensus on rating quality of evidence and strength of recommendations. BMJ. 2008;336:924-6. https://doi.org/10.1136/bmj. 39489.470347.AD.

7. Rosenbaum SE, Glenton C, Nylund HK, Oxman AD. User testing and stakeholder feedback contributed to the development of understandable and useful Summary of Findings tables for Cochrane reviews. J Clin Epidemiol. 2010;63:607-19. https://doi. org/10.1016/j.jclinepi.2009.12.013.

8. Downie LE, Makrai E, Bonggotgetsakul Y, et al. Appraising the quality of systematic reviews for age-related macular degeneration interventions: a systematic review. JAMA Ophthalmol. 2018; https://doi.org/10.1001/jamaophthalmol.2018.2620.

9. Ioannidis JP. The mass production of redundant, misleading, and conflicted systematic reviews and meta-analyses. Milbank Q. 2016;94:485-514. https://doi.org/10.1111/1468-0009.12210

10. Michelessi M, Lucenteforte E, Oddone F, et al. Optic nerve head and fibre layer imaging for diagnosing glaucoma. The Cochrane database of systematic reviews 2015:CD008803; https://doi.org/ 10.1002/14651858.CD008803.pub2.

11. Wallace BC, Noel-Storr A, Marshall IJ, Cohen AM, Smalheiser NR, Thomas J. Identifying reports of randomized controlled trials (RCTs) via a hybrid machine learning and crowdsourcing approach. J Am Med Inform Assoc: JAMIA. 2017;24:1165-68. https://doi.org/10.1093/jamia/ocx053.

12. Evans JR, Lawrenson JG. Antioxidant vitamin and mineral supplements for slowing the progression of age-related macular degeneration. Cochrane Database Syst Rev. 2017;7:CD000254; https://doi.org/10.1002/14651858.CD000254.pub4.

13. Li T, Ervin AM, Scherer R, Jampel H, Dickersin K. Setting priorities for comparative effectiveness research: a case study using primary open-angle glaucoma. Ophthalmology. 2010;117:1937-45. https://doi.org/10.1016/j.ophtha.2010.07.004.

14. Li T, Vedula SS, Scherer R, Dickersin K. What comparative effectiveness research is needed? A framework for using guidelines and systematic reviews to identify evidence gaps and 
research priorities. Ann Intern Med. 2012;156:367-77. https://doi. org/10.7326/0003-4819-156-5-201203060-00009.

15. Le JT, Hutfless S, Li T, et al. Setting Priorities for Diabetic Retinopathy Clinical Research and Identifying Evidence Gaps. Ophthalmology. Retina. 2017;1:94-102. https://doi.org/10.1016/j. oret.2016.10.003.

16. Saldanha IJ, Dickersin K, Hutfless ST, Akpek EK. Gaps in Current Knowledge and Priorities for Future Research in Dry Eye. Cornea. 2017;36:1584-91. https://doi.org/10.1097/ICO. 0000000000001350.

17. Lindsley K, Li T, Ssemanda E, Virgili G, Dickersin K. Interventions for Age-Related Macular Degeneration: Are Practice Guidelines Based on Systematic Reviews? Ophthalmology. 2016;123:884-97. https://doi.org/10.1016/j.ophtha.2015.12.004.

18. Mayo-Wilson E, Ng SM, Chuck RS, Li T. The quality of systematic reviews about interventions for refractive error can be improved: a review of systematic reviews. BMC Ophthalmol. 2017;17:164 https://doi.org/10.1186/s12886-017-0561-9.

19. Golozar A, Chen Y, Lindsley $\mathrm{K}$, et al. Identification and Description of Reliable Evidence for 2016 American Academy of Ophthalmology Preferred Practice Pattern Guidelines for Cataract in the Adult Eye. JAMA Ophthalmol. 2018;136:514-23. https:// doi.org/10.1001/jamaophthalmol.2018.0786.

20. Scherer RW, Sieving PC, Ervin AM, Dickersin K. Can we depend on investigators to identify and register randomized controlled trials? PLoS One. 2012;7:e44183 https://doi.org/10.1371/journal. pone. 0044183 .

21. Scherer RW, Huynh L, Ervin AM, Dickersin K. Using ClinicalTrials.gov to supplement information in ophthalmology conference abstracts about trial outcomes: a comparison study. PLoS One. 2015;10:e0130619 https://doi.org/10.1371/journal.pone. 0130619.

22. Scherer RW, Huynh L, Ervin AM, Taylor J, Dickersin K. ClinicalTrials.gov registration can supplement information in abstracts for systematic reviews: a comparison study. BMC Med Res Methodol. 2013;13:79 https://doi.org/10.1186/1471-2288-13-79.

23. Li T, Vedula SS, Hadar N, Parkin C, Lau J, Dickersin K. Innovations in data collection, management, and archiving for systematic reviews. Ann Intern Med. 2015;162:287-94. https://doi. org/10.7326/M14-1603.

24. Korevaar DA, Cohen JF, de Ronde MW, Virgili G, Dickersin K, Bossuyt PM. Reporting weaknesses in conference abstracts of diagnostic accuracy studies in ophthalmology. JAMA Ophthalmol. 2015;133:1464-7. https://doi.org/10.1001/jamaophthalmol. 2015.3577.

25. Saldanha IJ, Dickersin K, Wang X, Li T. Outcomes in Cochrane systematic reviews addressing four common eye conditions: an evaluation of completeness and comparability. PLoS One. 2014;9: e109400 https://doi.org/10.1371/journal.pone.0109400.

26. Saldanha IJ, Lindsley K, Do DV, et al. Comparison of Clinical Trial and Systematic Review Outcomes for the 4 Most Prevalent Eye Diseases. JAMA Ophthalmol. 2017;135:933-40. https://doi. org/10.1001/jamaophthalmol.2017.2583.

27. Law A, Lindsley K, Rouse B, Wormald R, Dickersin K, Li T. Missed opportunity from randomised controlled trials of medical interventions for open-angle glaucoma. $\mathrm{Br} \mathrm{J}$ Ophthalmol. 2017;101:1315-17. https://doi.org/10.1136/bjophthalmol-2016309695 .

28. Le JT, Viswanathan S, Tarver ME, Eydelman M, Li T. Assessment of the Incorporation of Patient-Centric Outcomes in Studies of Minimally Invasive Glaucoma Surgical Devices. JAMA Ophthalmol. 2016;134:1054-6. https://doi.org/10.1001/jamaophtha lmol.2016.2101.

29. Saldanha IJ, Petris R, Han G, Dickersin K, Akpek EK Research Questions and Outcomes Prioritized by Patients With Dry Eye. JAMA Ophthalmol. 2018. https://doi.org/10.1001/jamaophtha lmol.2018.3352.

30. Wang X, Hawkins BS, Dickersin K. Cochrane systematic reviews and co-publication: dissemination of evidence on interventions for ophthalmic conditions. Syst Rev. 2015;4:118 https://doi.org/10. 1186/s13643-015-0104-5.

31. Li T, Lindsley K, Rouse B, et al. Comparative Effectiveness of First-Line Medications for Primary Open-Angle Glaucoma: A Systematic Review and Network Meta-analysis. Ophthalmology. 2016;123:129-40. https://doi.org/10.1016/j.ophtha.2015.09.005.

32. Rouse B, Cipriani A, Shi Q, Coleman AL, Dickersin K, Li T. Network Meta-analysis for Clinical Practice Guidelines: A Case Study on First-Line Medical Therapies for Primary Open-Angle Glaucoma. Ann Intern Med. 2016;164:674-82. https://doi.org/10. 7326/M15-2367.

33. Ramke J, Evans JR, Gilbert CE Reducing inequity of cataract blindness and vision impairment is a global priority, but where is the evidence? BJO 2018 https://doi.org/10.1136/bjophthalmol2018-311985. 